\title{
AN INVESTIGATION INTO INTUITIONISTIC LOGIC WITH IDENTITY
}

\begin{abstract}
We define Kripke semantics for propositional intuitionistic logic with Suszko's identity ( $\mathrm{ISCl})$. We propose sequent calculus for $\mathrm{ISCl}$ along with cut-elimination theorem. We sketch a constructive interpretation of Suszko's propositional identity connective.
\end{abstract}

Keywords: non-Fregean logics, intuitionistic logic, admissibility of cut, propositional identity, congruence.

\section{Introduction}

In this paper we propose a constructive interpretation of Suszko's propositional identity operator $[10,1]$ along with a sequent calculus for the logic ISCI. The name 'ISCI' was introduced in [5]; however, already in [1] the authors, Bloom and Suszko, noted that $\mathrm{SCl}$ can be modified by taking intuitionistic logic as a base. ISCI is an extension of the propositional intuitionistic logic by a set of axioms which characterizes propositional identity operator ' $\approx$ '.

The strongest connective of classical propositional logic that may be used to express sameness of situations is the equivalence connective. But

* Corresponding author. During the work on this paper the first author benefited from the financial support of National Science Centre, Poland, grant no. 2015/17/B/HS1/02232.

** The work of the second author was supported financially by National Science Centre, Poland, grant no. 2017/26/E/HS1/00127. 
the question of equivalence of two formulas reduces to the question whether the formulas have the same logical value. This is not the case with the propositional identity - two formulas may be equivalent yet not identical in Suszko's sense. The philosophical motivation behind SCI was related to the ontology of situations - in classical logic, there are only two situations: Truth and Falsity, and the Truth (Falsity) is described by any true (false) proposition. According to Suszko, this is unfortunate, and could be remedied by allowing new identity connective, which describes the fact that two propositions describe the same situation. From this point of view, $\mathrm{SCl}$ can be considered as a generalization of classical logic in which we assume that there are at least two different situations.

The language of intuitionistic propositional logic also has the equivalence connective, and we can ask, again, whether the connective is suitable to express sameness of situations. In intuitionistic terms, we are not interested in propositions being true or false but in constructions which prove them. Equivalence of two formulas, $A$ and $B$, means that whenever $A$ is provable, $B$ is provable as well, and vice versa. But we can still think of a stronger notion which says that the classes of constructions proving $A$ and $B$ are exactly the same. As we shall see, this is the intended interpretation of the identity connective on the grounds of intuitionistic logic. Thus also in the intuitionistic setting, the identity connective gains an interpretation stronger than that of equivalence.

\section{Intuitionistic logic and Suszko's identity}

\subsection{BHK-interpretation and propositional identity}

Here is a version of the BHK-interpretation of logical constants. The last row depicts the first author's original interpretation of the propositional identity connective in constructive environment.

there is no proof of $\perp$ $a$ is a proof of $A \wedge B$ $a$ is a proof of $A \vee B$

$a=\left(a_{1}, a_{2}\right) ; a_{1}$ is a proof of $A$ and $a_{2}$ is a proof of $B$ $a=\left(a_{1}, a_{2}\right) ; a_{1}=0$ and $a_{2}$ is a proof of $A$ or $a_{1}=1$ and $a_{2}$ is a proof of $B$ 
$a$ is a proof of $A \supset B$

$a$ is a proof of $A \approx B$ $a$ is a construction that converts each proof $a_{1}$ of $A$ into a proof $a_{2}\left(a_{1}\right)$ of $B$ $a$ is a construction which shows that the classes of proofs of $A$ and $B$ are the same

In the present context a formula $A$ may be thought of as an expression which represents the set of its own proofs. If $A$ is identical to $B$, then $A$ and $B$ represent the same set. A construction, which can be used in establishing that the classes of proofs of two formulas are the same is the identity function $\lambda x . x$. We do not claim, however, that the identity function is the only object belonging to the identity type, but only that it is a natural choice to use this construction to intuitively validate identity axioms.

\subsection{Hilbert-style system for ISCI}

The language $\mathcal{L}_{\mathrm{ISCl}}$ of the logic ISCI is defined by the following grammar:

$$
A::=V|\perp| A \wedge A|A \vee A| A \supset A \mid A \approx A
$$

where $V$ is a denumerable set of propositional variables. Intuitionistic negation ' $\sim A$ ' is defined as ' $A \supset \perp$ '. Sometimes we will call formulas of the form ' $A \approx B$ ' equations. The axiom system for $\mathrm{ISCl}$ is obtained from any such system for INT, for example that from Table 1 (quoted after [4]) by the addition of $\approx$-specific axioms following under the four schemes $\left(\approx_{1}\right)-\left(\approx_{4}\right)$, where $\otimes \in\{\wedge, \vee, \supset, \approx\}$.

The presented axiom system for ISCI is called ' $\mathrm{H}_{\mathrm{ISCl}}$ '. By ' $S \vdash_{\mathrm{H}_{\mathrm{ISCl}}} A$ ' we mean that $A$ is derivable in $\mathrm{H}_{\mathrm{ISCl}}$ by means of axioms and formulas in $S$, where derivability is understood in a standard manner. If $\emptyset \vdash_{\mathrm{H}_{\mathrm{SCl}}} A$, then we will say that $A$ is a thesis of $\mathrm{H}_{\mathrm{ISCl}}$. (Here is an example of a thesis of $\mathrm{H}_{\mathrm{ISCl}}$ other than an axiom: $\perp \supset \perp$. We shall use it in Lemma 3.)

If $A \approx B$ holds, we will say that $A$ and $B$ are identical. By the symbol $|A|$ we denote the class of proofs of $A$. Let us note that axioms of Suszko's identity are valid under the interpretation proposed in Subsection 1.1.

$\left(\approx_{1}\right)$ Naturally, $|A|=|A|$.

$\left(\approx_{2}\right)$ Assume that $|A|=|B|$. In this case, a construction that converts any element of $|A|$ into a proof of $\perp$ is a construction that converts any element of $|B|$ into a proof of $\perp$. Therefore there exists a function which transforms each proof of the identity of $A$ and $B$ into a proof of the identity of $A \supset \perp$ and $B \supset \perp$. 
Table 1. Axioms of intuitionistic logic INT

$$
\begin{aligned}
& \mathbf{H}_{1} \quad A \supset(B \supset A) \\
& \mathbf{H}_{2}(A \supset B) \supset((A \supset(B \supset C)) \supset(A \supset C)) \\
& \mathbf{H}_{3} \quad A \supset(B \supset(A \wedge B)) \\
& \mathbf{H}_{4}(A \wedge B) \supset A \\
& \mathbf{H}_{5}(A \wedge B) \supset B \\
& \mathbf{H}_{6}(A \supset C) \supset((B \supset C) \supset((A \vee B) \supset C)) \\
& \mathbf{H}_{7} A \supset(A \vee B) \\
& \mathbf{H}_{8} B \supset(A \vee B) \\
& \mathbf{H}_{9}(A \supset B) \supset((A \supset \sim B) \supset \sim A) \\
& \mathbf{H}_{10} \sim A \supset(A \supset B) \\
& \text { MP from } A \text { and } A \supset B \text { conclude } B \\
& \left(\approx_{1}\right) A \approx A \\
& \left(\approx_{2}\right)(A \approx B) \supset((A \supset \perp) \approx(B \supset \perp)) \\
& \left(\approx_{3}\right)(A \approx B) \supset(B \supset A) \\
& \left(\approx_{4}\right)((A \approx B) \wedge(C \approx D)) \supset((A \otimes C) \approx(B \otimes D))
\end{aligned}
$$

$\left(\approx_{3}\right)$ If $A$ is identical to $B$, then each proof of $B$ can be transformed (by the identity function $\lambda x . x$ ) into a proof of $A$. Therefore there is a function which transforms each proof of the identity of $A$ and $B$ into a proof of $B \supset A$ (and $A \supset B$, but this is implied by the other conditions).

$\left(\approx_{4}\right)$ We shall argue that each pair $\left(a_{1}, a_{2}\right)$, where $a_{1}$ is a proof of the identity of $A$ and $B$ and $a_{2}$ is a proof of the identity of $C$ and $D$, can be transformed into a proof of the identity of $A \otimes C$ and $B \otimes D$. Assume $|A|=|B|$ and $|C|=|D|$ and:

(a) $\otimes=\wedge$. If $a_{1}$ is a proof of $A$ and $a_{2}$ is a proof of $C$, then, by assumption, pair $\left(a_{1}, a_{2}\right)$ constitutes a proof of $B$ and $D$; and vice versa: if a pair proves $B$ and $D$, then it proves $A$ and $C$, respectively. It follows that $|A| \times|C|=|B| \times|D|$.

(b) $\otimes=\vee$. Let $\left(0, a_{2}\right)$ be a proof of $A \vee C$ (thus $a_{2}$ is a proof of $A$ ). Since $|A|=|B|,\left(0, a_{2}\right)$ is also a proof of $B \vee D$. For a similar reason, if $\left(1, a_{2}\right)$ is a proof of $A \vee C$, then it is also a proof of $B \vee D$. And vice versa: from $B \vee D$ to $A \vee C$. 
(c) $\otimes=\supset$. Assume $\lambda x . y$ is a proof of $A \supset C$. Since $|A|=|B|$ and $|C|=|D|$ this function is also a proof of $B \supset D$ (and vice versa).

(d) $\otimes=\approx$. Assume $\lambda x . x$ is a proof of $A \approx C$. Since $|A|=|B|$ and $|C|=|D|$ this function also proves $B \approx D$.

The identity of formulas $A$ and $B$ amounts to the existence of a function showing the identity of sets $|A|$ and $|B|$. Let us note that according to the proposed interpretation, the identity connective is stronger than intuitionistic equivalence. If $A$ and $B$ are identical, then they are intuitionisticaly equivalent (that is, every proof of $A$ can be transformed into a proof of $B$ and vice versa). But the converse does not hold. From the fact that $A$ and $B$ are intuitionisticaly equivalent one cannot derive the conclusion that the function which converts proofs of $A$ into proofs of $B$ is the identity function $\lambda x . x$ between $|A|$ and $|B|$.

In type-theoretical terms $[11,3,2]$, a formula $A \supset B$ corresponds to the type of functions which take arguments of the type $A$ and return values of type $B$

$$
\left(\lambda x^{A} \cdot t^{B}\right)^{A \supset B},
$$

whereas a type $A \approx B$ is certainly inhabited by identity functions

$$
\left(\lambda x^{A} \cdot x^{B}\right)^{A \approx B} .
$$

Note that the set of all functions of the type $A \approx B$ is a subset of the set of all functions of the type $A \supset B$; each function of the type $A \approx B$ is also of the type $A \supset B$. Let us stress once again that identity is stronger than intuitionistic equivalence. This point becomes clear if we realise that an equation $A \approx B$ is a thesis of $\mathrm{ISCl}$ if and only if it represents a function: $\left(\lambda x^{A} \cdot x^{A}\right)^{A \approx A}$, i.e., when ' $A$ ' and ' $B$ ' is the same formula.

\subsection{Semantics}

An algebraic semantic for $\mathrm{ISCl}$ is given in [5] along with a sketch of completeness proof. Here we propose a simple semantic approach based on Kripke frames.

DEFINITION 1 (ISCI frame). By an $\mathrm{ISCl}$ frame we mean an ordered pair $\boldsymbol{F}=\langle W, \leq\rangle$, where $W$ is a non-empty set and $\leq$ is a reflexive and transitive binary relation on $W$. 
By 'For ${ }_{0}$ ' we shall mean the sum of $V$ (the set of all variables) and the set of all equations. If $\boldsymbol{F}=\langle W, \leq\rangle$ is an ISCI frame, then by assignment in $\boldsymbol{F}$ we mean a function:

$$
v: \text { For }_{0} \times W \longrightarrow\{0,1\} .
$$

An assignment is called $\mathbf{I S C l}$-admissible, provided that for each $w \in W$, and for arbitrary formulas $A, B, C, D:$ (1) $v(A \approx A, w)=1$, (2) if $v(A \approx B, w)=1$, then $v((A \supset \perp) \approx(B \supset \perp), w)=1$, and $(3)$ if $v(A \approx$ $B, w)=1$ and $v(C \approx D, w)=1$, then $v((A \otimes C) \approx(B \otimes D), w)=1$. The three conditions capture $\approx$-specific axioms falling under $\left(\approx_{1}\right),\left(\approx_{2}\right),\left(\approx_{4}\right)$, respectively. The third scheme will be captured in the notion of forcing.

DEFINITION 2 (Forcing). Let $v$ be an $\mathrm{ISCl}$-admissible assignment in a given frame $\boldsymbol{F}$. A forcing relation $\Vdash$ determined by $v$ in $\boldsymbol{F}$ is a relation between elements of $W$ and elements of $\mathcal{L}_{\mathrm{ISCl}}$ which satisfies, for arbitrary $w \in W$, the following conditions:

(1) $\quad w \Vdash p_{i}$ iff $v\left(p_{i}, w\right)=1$;

(2) $w \nVdash \perp$;

(3) $w \Vdash A \wedge B$ iff $w \Vdash A$ and $w \Vdash B$;

(4) $w \Vdash A \vee B$ iff $w \Vdash A$ or $w \Vdash B$;

(5) $w \Vdash A \supset B$ iff for each $w^{\prime}$ such that $w \leq w^{\prime}$, if $w^{\prime} \Vdash A$ then $w^{\prime} \Vdash B$;

(mon) if $w \Vdash p_{j}$ and $w \leq w^{\prime}$, then $w^{\prime} \Vdash p_{j}$;

$\left(\right.$ mon $\left._{\approx}\right)$ if $w \Vdash A \approx B$ and $w \leq w^{\prime}$, then $w^{\prime} \Vdash A \approx B$;

$(\approx) \quad$ if $w \Vdash A \approx B$, then $w \Vdash B \supset A$.

Note that the condition (mon) can be strengthened to:

$\left(m o n^{\prime}\right) \quad$ where $A$ is not an equation, if $w \Vdash A$ and $w \leq w^{\prime}$, then $w^{\prime} \Vdash A$,

and combined with $\left(\operatorname{mon}_{\approx}\right)$, the conditions yield monotonicity for all formulas of $\mathcal{L}_{\mathrm{ISCl}}$.

Definition 3. An ISCI model is a triple $\boldsymbol{M}=\langle W, \leq, \Vdash\rangle$, where $\boldsymbol{F}=\langle W, \leq\rangle$ is an $\mathrm{ISCl}$ frame and $\Vdash$ is a forcing relation determined by some $\mathrm{ISCl}$ admissible assignment in $\boldsymbol{F}$.

A formula $A$ which is forced by every world of an $\mathrm{ISCl}$ model, that is, such that $w \Vdash A$ for each $w \in W$, is called true in the model.

A formula true in every $\mathrm{ISCl}$ model is called $\mathrm{ISCl}-$ valid. 
TheOrem 1 (soundness). Let $A$ be a formula of $\mathcal{L}_{\mathrm{ISCl}}$. If $\emptyset \vdash_{\mathrm{H}_{\mathrm{ISCl}}} A$, then $A$ is $\mathrm{ISCl}$-valid.

Proof: Since the semantics for ISCI is based on Kripke semantics for INT, we omit most of the proof. One fact worth noting is that showing that axioms $\mathbf{H}_{1}$ and $\mathbf{H}_{3}$ are ISCl-valid requires not only (mon), but $\left(m_{0} n_{\approx}\right)$ as well. The argument for $\mathbf{H}_{1}$ goes as follows. Assume that $\mathbf{H}_{1}: A \supset(B \supset A)$ is not true in some ISCI model $\boldsymbol{M}=\langle W, \leq, \Vdash\rangle$. Then there is $w \in W$ such that: $w \forall A \supset(B \supset A)$. Hence, there is a world $w^{1}$ available from $w$, such that $w^{1} \Vdash A$, but $w^{1} \Vdash B \supset A$. Again, there is a world $w^{2}$ visible from $w^{1}$ such that $w^{2} \Vdash B$ and $w^{2} \forall A$. But since we have proved $A$ at $w^{1}$ and the next step is $w^{2}, A$ is also proved at $w^{2}$ (monotonicity), which results in a contradiction. However, in the last sentence we cannot rely on (mon) only, since $A$ can be an equation.

Further, the three conditions in the definition of ISCl-admissible assignment and condition $(\approx)$ in the definition of forcing warrant that the $\approx-$ specific axioms are ISCl-valid. Needless to say, MP preserves ISCl-validity, therefore each thesis of $\mathrm{H}_{\mathrm{ISCl}}$ is ISCl-valid.

\subsection{Discussion}

Independently of the solutions which we have adapted in the previous subsection, it seems worth to consider the idea of monotonicity of identity, i.e., the condition:

$\left(\right.$ mon $\left._{\approx}\right) \quad$ if $w \Vdash A \approx B$ and $w \leq w^{\prime}$, then $w^{\prime} \Vdash A \approx B$.

First of all, $(m o n \approx)$ does not follow by induction from $(m o n)$. The reason is that the components of identity may be both true at a given world, but a formula which states their identity may be false. Hence there are two possibilities which are worth considering:

1 . We accept $(m o n \approx)$, as we did above. There is a good reason for that from the intuitionistic viewpoint. A proof of each formula should be remembered, i.e., if it has been proved at a given point, then it should also be provable at a later point. According to this interpretation also a proved equation $A \approx B$ remains proved, irrespective of the proofs of $A$ and/or $B$ available at further points (further in the sense of $\leq$ ).

2 . We reject $\left(m o n_{\approx}\right)$. One can find good reasons for rejecting $\left(m o n_{\approx}\right)$; under the BHK-interpretation the truth of $A \approx B$ yields the existence 
of a construction which shows that the classes of proofs of $A$ and $B$ are the same. But when we move ahead along with $\leq$, some new proofs of $A$ and/or of $B$ can be found, and then the classes of the appropriate proofs $|A|$ and $|B|$ may become distinct. Hence the connective of constructive identity without $\left(m_{0} \approx\right)$ seems more adequate to account for provability as acquired by a human being.

However, rejecting $(m o n \approx)$ yields some serious consequences. As shown in the proof of Theorem 1 , in the presented setting $\left(m_{0} \approx\right)$ is necessary to prove that axioms $\mathbf{H}_{1}$ and $\mathbf{H}_{3}$ are ISCl-valid. Hence if $(m o n \approx)$ is rejected, one needs to warrant the validity of the axioms in some other way.

One the other hand, let us observe that $\left(\operatorname{mon}_{\approx}\right)$ is neither necessary nor sufficient in proving that the $\approx$-specific axioms are ISCl-valid. In the presented setting this is warranted in the notion of ISCl-admissibility and in the additional condition $(\approx)$ that forcing must preserve.

\subsection{Completeness of Hilbert-style system for $\mathrm{ISCl}$}

Here we give a Henkin-style completeness proof of $\mathrm{H}_{\mathrm{ISCl}}$ with respect to the presented semantics.

Let $S$ and $F$ stand for a set of formulas and a single formula of $\mathcal{L}_{\mathrm{ISCl}}$, respectively. We will say that $S$ is $F$ - $\mathrm{H}_{\mathrm{ISCl}}$-consistent iff $S \forall_{\mathrm{H}_{\mathrm{ISCl}}} F$; otherwise $S$ is called $F-\mathrm{H}_{\mathrm{ISCl}}$-inconsistent. $S$ is called maximally $F-\mathrm{H}_{\mathrm{ISCl}}$-consistent iff it is $F-\mathrm{H}_{\mathrm{ISCl}}-$-consistent and no proper superset of $S$ is $F-\mathrm{H}_{\mathrm{ISCl}}-$ consistent.

For simplicity, we will write ' $F$-(in)consistent' instead of ' $F$ - $\mathrm{H}_{\mathrm{ISCl}}$-(in)consistent'.

Lemma 1 (Lindenbaum's lemma). Let $F$ stand for a formula of $\mathcal{L}_{\mathrm{ISCl}}$. For every $F$-consistent set $S$ there is a maximally $F$-consistent set $\mathbf{S} \supseteq S$.

Proof: Let us recall the well-known construction. We enumerate all formulas of $\mathcal{L}_{\mathrm{ISCl}}$ :

$$
B_{1}, B_{2}, \ldots, B_{n}, \ldots
$$

Suppose that $S$ is an $F$-consistent set, that is, $S \nvdash_{\mathrm{H}_{\text {ISCI }}} F$. We construct an infinite sequence of sets by means of the following rules:

$$
S_{0}=S
$$




$$
S_{n+1}= \begin{cases}S_{n} & \text { if } S_{n} \cup\left\{B_{n+1}\right\} \vdash_{\mathrm{H}_{\mathrm{ISCl}}} F \\ S_{n} \cup\left\{B_{n+1}\right\} & \text { otherwise. }\end{cases}
$$

It follows from the construction that each member of this sequence is $F$ consistent and the set $\mathbf{S}=\bigcup_{n=0}^{\infty} S_{n}$ is maximally $F$-consistent.

If for some formula $F$, a set $S$ is (maximally) $F$-consistent, and the formula is irrelevant in a given context, then we will say simply that $S$ is (maximally) consistent. Let us now prove:

LEMma 2. A formula of language $\mathcal{L}_{\mathrm{ISCl}}$ is a thesis of $\mathrm{H}_{\mathrm{ISCl}}$ if and only if it is an element of each maximally consistent set.

Proof: Assume that: (a) it is the case that $\emptyset \vdash_{\mathrm{H}_{\text {IsCl }}} A$, but there is a maximally consistent set $S$ such that (b) $A \notin S$. By definition, there is some formula $F$ such that $S \nvdash \mathrm{H}_{\mathrm{ISCl}} F$ (that is, for some $F, S$ is $F$-consistent), and by the construction of $S$ and by (b), for some $S_{n} \subseteq S, S_{n} \cup\{A\} \vdash_{\mathrm{H}_{\mathrm{ISCl}}} F$. Hence, and by deduction theorem, $S_{n} \vdash_{\mathrm{H}_{\mathrm{ISCl}}} A \supset F$, and since (a) yields $S_{n} \vdash_{\mathrm{H}_{\text {ISCl }}} A$ (weakening), also $S_{n} \vdash_{\mathrm{H}_{\mathrm{ISCl}}} F$ (by MP). But then $S \supseteq S_{n}$ is not $F$-consistent. A contradiction.

For the only-if part assume that $A$ is not a thesis of $\mathrm{H}_{\mathrm{ISCl}}$, that is, $\emptyset H_{\mathrm{H}_{\text {ISCI }}} A$. It follows that the empty set is $A$-consistent. Thus, by Lemma 1 , there is a maximally $A$-consistent set $\mathbf{S} \supseteq \emptyset$, and hence $A \notin \mathbf{S}$.

Lemma 3. Let $\mathbf{S}$ be a maximally consistent set. The following conditions are satisfied:

1. $\perp \notin \mathbf{S}$;

2. $A \in \mathbf{S}$ iff $\mathbf{S} \vdash_{\mathrm{H}_{\mathrm{ISCl}}} A$;

3. $A \wedge B \in \mathbf{S}$ iff $A \in \mathbf{S}$ and $B \in \mathbf{S}$;

4. $A \vee B \in \mathbf{S}$ iff $A \in \mathbf{S}$ or $B \in \mathbf{S}$;

5. if $A \supset B \in \mathbf{S}$ and $A \in \mathbf{S}$, then $B \in \mathbf{S}$;

6. if $A \supset B \notin \mathbf{S}$, then $\mathbf{S} \cup\{A\}$ is B-consistent.

Proof: Suppose that $\mathbf{S}$ is a maximally consistent set. Then for some formula $F$, (a) $\mathbf{S} \nvdash_{\mathrm{H}_{\text {Iscl }}} F$.

(ad1) If $\perp \in \mathbf{S}$, then for each formula, in particular for $F$, we have $\mathbf{S} \vdash_{\mathrm{H}_{\text {Iscl }}}$ $F,{ }^{1}$ which contradicts (a). Hence $\perp \notin \mathbf{S}$.

\footnotetext{
${ }^{1} \mathrm{~A}$ justification is such that: $(\perp \supset \perp) \supset(\perp \supset F)$ is an instance of $\mathbf{H}_{10}$. Moreover, $\mathbf{S} \vdash_{\mathrm{H}_{\mathrm{ISCl}}} \perp \supset \perp$, thus $\mathbf{S} \vdash_{\mathrm{H}_{\mathrm{ISCl}}} \perp \supset F$ and $\mathbf{S} \vdash_{\mathrm{H}_{\mathrm{ISCl}}} F$ by $\mathbf{M P}$.
} 
(ad2) The if-then direction holds by reflexivity of $\vdash_{\mathrm{H}_{\mathrm{ISCl}}}$. For the only-if part assume that $A \notin \mathbf{S}$. Then $\mathbf{S} \cup\{A\} \vdash_{\mathrm{H}_{\mathrm{ISCl}}} F$ by the construction of a maximally consistent set, and hence also $\mathbf{S} \vdash_{\mathrm{H}_{\text {Iscl }}} A \supset F$ (deduction theorem). If, in addition, we assumed that $\mathbf{S} \vdash_{\mathrm{H}_{\mathrm{ISCl}}} A$, then we would obtain $\mathbf{S} \vdash_{\mathrm{H}_{\mathrm{ISCl}}} F$ by $\mathbf{M P}$, therefore $\mathbf{S} \nvdash_{\mathrm{H}_{\mathrm{ISCl}}} A$.

(ad3) For the if-then part assume that $A \wedge B \in \mathbf{S}$. Then $\mathbf{S} \vdash_{\mathrm{H}_{\mathrm{ISCl}}} A \wedge B$; also $\mathbf{S} \vdash_{\mathrm{H}_{\mathrm{ISCl}}}(A \wedge B) \supset A$, since the formula is an axiom of $\mathrm{H}_{\mathrm{ISCl}}$. Thus $\mathbf{S} \vdash_{\mathrm{H}_{\text {ISCl }}} A$ by $\mathbf{M P}$, and, by clause 2 of this lemma, $A \in \mathbf{S}$. The reasoning is similar for $B \in \mathbf{S}$.

For the only-if direction assume that $A \in \mathbf{S}$ and $B \in \mathbf{S}$. Then $\mathbf{S} \vdash_{\mathrm{H}_{\mathrm{ISCl}}} A$ and $\mathbf{S} \vdash_{\mathrm{H}_{\mathrm{ISCl}}} B$. By using axiom $\mathbf{H}_{3}$ and $\mathbf{M P}$ we get $\mathbf{S} \vdash_{\mathrm{H}_{\text {ISCl }}} A \wedge B$, and finally $A \wedge B \in \mathbf{S}$ by clause 2 .

(ad4) If-then direction: if $A \notin \mathbf{S}$ and $B \notin \mathbf{S}$, then $\mathbf{S} \vdash_{\mathrm{H}_{\mathrm{Iscl}}} A \supset F$ and $\mathbf{S} \vdash_{\mathrm{H}_{\text {Iscl }}} B \supset F$. Then by $\mathbf{H}_{6}$ and $\mathbf{M P}$ we have $\mathbf{S} \vdash_{\mathrm{H}_{\text {Iscl }}} A \vee B \supset F$. By (a) and clause $2, A \vee B \notin \mathbf{S}$.

For the only-if side assume that $A \in \mathbf{S}$, but $A \vee B \notin \mathbf{S}$. Using clause 2, deduction theorem, axiom $\mathbf{H}_{7}$ and $\mathbf{M P}$, one arrives at $\mathbf{S} \vdash_{\mathrm{H}_{\mathrm{ISCl}}} F$. Thus $A \vee B \in \mathbf{S}$. The reasoning is similar if $B \in \mathbf{S}$.

(ad5) By clause 2 and MP.

(ad6) If $A \supset B \notin \mathbf{S}$, then, by clause $2, \mathbf{S} \nvdash \mathrm{H}_{\text {ISCl }} A \supset B$. Hence also $\mathbf{S} \cup\{A\} \nvdash_{\mathrm{H}_{\mathrm{ISCl}}} B$ (deduction theorem). In other words, $\mathbf{S} \cup\{A\}$ is $B$-consistent.

Definition 4 (Canonical ISCI model). Canonical ISCI model is a triple $\boldsymbol{M}=\left\langle W, \subseteq_{W}, \in_{W}\right\rangle$, where $W$ is the set of all maximally consistent sets of formulas of $\mathcal{L}_{\mathrm{ISCl}}, \subseteq_{W}$ is the set inclusion in $W$, and $\in_{W}$ is the membership relation between formulas of $\mathcal{L}_{\mathrm{ISCl}}$ and elements of $W$.

Frame $\left\langle W, \subseteq_{W}\right\rangle$ is an ISCl-frame, because, first, $W$ is non-empty (the empty set is a consistent set, and by Lemma 1, it has a maximally consistent superset, hence at least one maximally consistent set exists), and the set inclusion $\subseteq_{W}$ is reflexive and transitive. We still need to show that the canonical ISCl model is in fact an ISCI model.

Lemma 4. The canonical ISCI model satisfies Definition 3, that is, is an ISCI model.

Proof: Let $\boldsymbol{M}=\left\langle W, \subseteq_{W}, \in_{W}\right\rangle$ be the canonical ISCI model. We already know that the structure $\left\langle W, \subseteq_{W}\right\rangle$ is an ISCl-frame. Hence what is left to show is that the membership relation $\epsilon_{W}$ satisfies Definition 2 of forcing. 
As the required assignment in $\left\langle W, \subseteq_{W}\right\rangle$ we take function $v: F o r_{0} \times$ $W \longrightarrow\{0,1\}$ defined: $v(A, w)=1$ iff $A \in w$. The assignment is ISCIadmissible, since each $\approx$-specific axiom belongs to each maximally consistent set by the definition of consistent sets (see clause 2 of Lemma 3). (We obtain conditions (2) and (3) defining ISCl-admissibility by MP.) Clearly, $\epsilon_{W}$ extends $v$, and thus it satisfies clause (1) of Definition 2. Clauses (2)-(4) hold by Lemma 3.

Clause (5), if-then direction: assume that $A \supset B \in_{W} w$, and that $w \subseteq_{W} w^{*}$; hence also $A \supset B \in_{W} w^{*}$. By clause 5 of Lemma 3, if $A \in_{W} w^{*}$, then also $B \in_{W} w^{*}$. Clause (5), only-if direction: suppose that $A \supset B \notin_{W}$ $w$. By clause 6 of Lemma 3, $w \cup\{A\}$ is $B$-consistent. By Lemma 1, there is a maximally $B$-consistent set $w^{*} \supseteq w \cup\{A\}$. It follows that $w \subseteq_{W} w^{*}$, $A \in_{W} w^{*}$ and $B \notin_{W} w^{*}$.

Monotonicity conditions $(m o n)$ and $\left(\right.$ mon $\left._{\approx}\right)$ hold trivially by the fact that the relation between worlds is set inclusion.

Finally, since each $\approx$-specific axiom, $\left(\approx_{3}\right):(A \approx B) \supset(B \supset A)$ in particular, belongs to each maximally consistent set, condition $(\approx)$ holds as well.

Let us note that if $A$ is a thesis of $\mathrm{H}_{\mathrm{ISCl}}$, then $A$ is an element of each maximally s-consistent set (Lemma 2), and thus $A$ is true in the canonical ISCl-model.

THEOREM 2 (completeness). If a formula is ISCI-valid, then it is a thesis of $\mathrm{H}_{\mathrm{ISCl}}$.

Proof: The proof is by contraposition. Assume that a formula is not a thesis of $\mathrm{H}_{\mathrm{ISCl}}$. Thus, by Lemma 2, there exists maximally consistent set $w$ such that $A \notin w$. Thus there is a world $w$ in the canonical $\mathbf{I S C l}$ model which does not contain $A$. Hence $A$ is not ISCl-valid.

\section{Sequent calculi for $\mid \mathrm{SCl}$}

\subsection{Axioms and rules}

There is a number of strategies of building sequent calculi or natural deduction systems for axiomatic theories based on a certain logic (see for example $[12,7,9,8])$. The strategy we are interested in enables one to turn each axiom of a given axiomatic system into a rule of a corresponding 
sequent calculus in such a way that all structural rules - the cut rule in particular - are admissible in the generated calculus. The strategy requires that the initial axioms, from which the rules will be generated, are of the form:

$$
P_{1} \wedge \ldots \wedge P_{m} \rightarrow Q_{1} \vee \ldots \vee Q_{n}
$$

where $P_{i}$ and $Q_{j}$ are propositional variables. Naturally, the specific $\approx$ axioms do not fit into this form. Thus we will generalize this strategy to axioms of the form:

$$
A_{1} \wedge \ldots \wedge A_{m} \rightarrow B_{1} \vee \ldots \vee B_{n}
$$

where $A_{i}, B_{j}$ are arbitrary formulas. The sequent rules corresponding to (2.2) should present as follows ( $\Gamma$ and $\Delta$ stand for multisets of formulas):

$$
\begin{aligned}
& \frac{B_{1}, A_{1}, \ldots, A_{m}, \Gamma \Rightarrow \Delta \quad \ldots \quad B_{n}, A_{1}, \ldots, A_{m}, \Gamma \Rightarrow \Delta}{A_{1}, \ldots, A_{m}, \Gamma \Rightarrow \Delta} L \\
& \frac{\Gamma \Rightarrow \Delta, B_{1}, \ldots, B_{n}, A_{1} \quad \ldots \quad \Gamma \Rightarrow \Delta, B_{1}, \ldots, B_{n}, A_{n}}{\Gamma \Rightarrow \Delta, B_{1}, \ldots, B_{n}} R
\end{aligned}
$$

If each axiom is transformed into a left (right) rule, then we obtain a left (right) system. Let us observe, however, that the right rule is problematic in the constructive setting, due to the usual restriction on the consequent of a sequent in intuitionistic logic. Sequents used in constructing the sequent calculus for ISCI will be of the form:

$$
\Gamma \Rightarrow A
$$

where $\Gamma$ is a finite, possibly empty, multiset of formulas of $\mathcal{L}_{\mathrm{ISCl}}$ and $A$ is a single formula of $\mathcal{L}_{\mathrm{ISCl}}$.

The restriction on the consequent of a sequent forces us to define only left system for ISCl. However, according to the presented strategy of rules construction, each left system constructed by means of this method needs to satisfy the following additional condition:

Definition 5 (Closure Condition, [6]). If a system with nonlogical rules has a rule, where a substitution instance in the atoms produces a rule of the form:

$$
\frac{B_{1}, A_{1}, \ldots, A_{m-2}, A, A, \Gamma \Rightarrow \Delta \quad \ldots \quad B_{n}, A_{1}, \ldots, A_{m-2}, A, A, \Gamma \Rightarrow \Delta}{A_{1}, \ldots, A_{m-2}, A, A, \Gamma \Rightarrow \Delta} R
$$


then it also has to contain the rule:

$$
\frac{B_{1}, A_{1}, \ldots, A_{m-2}, A, \Gamma \Rightarrow \Delta \quad \ldots \quad B_{n}, A_{1}, \ldots, A_{m-2}, A, \Gamma \Rightarrow \Delta}{A_{1}, \ldots, A_{m-2}, A, \Gamma \Rightarrow \Delta} R^{*}
$$

The closure condition ensures the existence of rules in a given system which are essential for the admissibility of contraction in that system.

Table 2. Structural rules

$$
\begin{gathered}
\frac{\Gamma \Rightarrow C}{A, \Gamma \Rightarrow C} L_{w} \quad \frac{A, A, \Gamma \Rightarrow C}{A, \Gamma \Rightarrow C} L_{c t r} \\
\frac{\Gamma^{\prime} \Rightarrow D \quad D, \Gamma^{\prime \prime} \Rightarrow C}{\Gamma^{\prime}, \Gamma^{\prime \prime} \Rightarrow C} c u t
\end{gathered}
$$

Sequent calculus $\mathbf{G} \mathbf{3}_{\mathrm{ISCl}}$ is composed of the structural rules displayed in Table 2, and the logical rules presented in Table 3.

Following the presented strategy, we obtain the $\approx$-specific (left) rules listed in Table 3. It is worth noticing that none of the rules is directly obtained from axiom $(\approx)_{2}$. In the absence of negation as a primitive connective, axiom $(\approx)_{2}$ is provable with the use of rules $L_{\approx}^{3}$ and $L_{\approx}^{1}$ (in a sense, it is a particular case of congruence). The rule $L_{\approx}^{3} *$ belongs to the system due to the closure condition.

Let $\mathbf{M}=\langle W, \leq, \Vdash\rangle$ be an ISCI model and $w \in W$. We will say that a sequent $\Gamma \Rightarrow C$ is satisfied at $w$ in $\boldsymbol{M}$ iff the fact that $w$ forces each member of $\Gamma$ implies that it also forces $C$. A sequent is said to be true in a model iff it is satisfied at each world in this model.

LEMma 5. Each rule of $\mathbf{G 3}_{\mathrm{ISCl}}$ preserves truth in an $\mathrm{ISCl}$ model.

Proof: Let us consider $L_{\approx}^{1}$ only. Let $\boldsymbol{M}=\langle W, \leq, \Vdash\rangle$ be an arbitrary model. Assume that sequent $A \approx A, \Gamma \Rightarrow C$ is true in $\boldsymbol{M}$ and that $\Gamma \Rightarrow C$ is not. Thus there exists a world $w$ such that $w$ forces each member of $\Gamma$, but $w \Vdash C$. Naturally $w \Vdash A \approx A$. Thus $A \approx A, \Gamma \Rightarrow C$ is not true in $\boldsymbol{M}$, contrary to our assumption. 
Table 3. Logical rules of $\mathbf{G} 3_{\mathrm{ISCl}}$

$$
\begin{array}{cc}
p_{i}, \Gamma \Rightarrow p_{i} & A \approx B, \Gamma \Rightarrow A \approx B \\
\frac{\perp, \Gamma \Rightarrow C}{\perp} L_{\perp} & \frac{\Gamma \Rightarrow A \quad \Gamma \Rightarrow B}{\Gamma \Rightarrow A \wedge B} R_{\wedge} \\
\frac{A, B, \Gamma \Rightarrow C}{A \wedge B, \Gamma \Rightarrow C} L_{\wedge} & \frac{\Gamma \Rightarrow B}{\Gamma \Rightarrow A \vee B} R_{\vee} \\
\frac{A, \Gamma \Rightarrow C \quad B, \Gamma \Rightarrow C}{A \vee B, \Gamma \Rightarrow C} L_{\vee} & \frac{A, \Gamma \Rightarrow B}{\Gamma \Rightarrow A \supset B} R_{\supset} \\
\frac{\Gamma \supset B, \Gamma \Rightarrow A \quad B, \Gamma \Rightarrow C}{\Gamma \Rightarrow A \vee B} R_{\vee} & \Rightarrow \frac{A \approx B, \Gamma \Rightarrow B \quad A, A \approx B, \Gamma \Rightarrow C}{A \supset B, \Gamma \Rightarrow C} L_{\approx}^{2} \\
\frac{A \approx A, \Gamma \Rightarrow C}{\Gamma \Rightarrow C} L_{\approx}^{1} & A \approx B, \Gamma \Rightarrow C \\
\frac{(A \otimes C) \approx(B \otimes D), A \approx B, C \approx D, \Gamma \Rightarrow E}{A \approx B, C \approx D, \Gamma \Rightarrow E} & L_{\approx}^{3} \\
\frac{(A \otimes A) \approx(B \otimes B), A \approx B, \Gamma \Rightarrow E}{A \approx B, \Gamma \Rightarrow E} &
\end{array}
$$

\subsection{Completeness}

The easiest way to show completeness of $\mathbf{G} \mathbf{3}_{\mathrm{ISCl}}$ is to prove that it can simulate axiomatic system.

First, the following is easy to show by induction on the structure of formula $A$ :

Corollary 1. For each formula $A$ of $\mathcal{L}_{\mathrm{ISCl}}$, sequent $A, \Gamma \Rightarrow A$ is provable in $\mathbf{G} \mathbf{3}_{\mathrm{ISCl}}$.

THEOREM 3. $\mathbf{G 3}_{\mathrm{ISCl}}+[$ cut $]$ is complete with respect to ISCI-semantics.

Proof: The Hilbert-style system $\mathrm{H}_{\mathrm{ISCl}}$ is complete with respect to the presented ISCl-semantics, as we have shown in the previous section. One 
can simulate $\mathrm{H}_{\mathrm{ISCl}}$ in $\mathbf{G} \mathbf{3}_{\mathrm{ISCl}}+[$ cut $]$. Each axiom is derivable - see for example $\approx_{3}:$

$$
\begin{gathered}
\frac{A \approx B, B \Rightarrow B \quad A, A \approx B, B \Rightarrow A}{\frac{A \approx B, B \Rightarrow A}{A \approx B \Rightarrow B \supset A} R_{\supset}} L_{\approx}^{2} \\
\frac{A \approx(A \approx B) \supset(B \supset A)}{\Rightarrow\left(B \supset{ }_{\supset}\right.}
\end{gathered}
$$

and, by Corollary 1, the following derivation proves that MP can be reconstructed in $\mathbf{G} \mathbf{3}_{\mathrm{ISCl}}+[$ cut $]$.

$$
\Rightarrow A \frac{\frac{A \supset B, A \Rightarrow A \quad B, A \Rightarrow B}{A \supset B, A \Rightarrow B}}{\Rightarrow A \supset B} L_{\supset}
$$

Thus $\mathbf{G} \mathbf{3}_{\mathrm{ISCl}}+[\mathrm{cut}]$ enable us to prove formulas in a similar manner to the one used in $\mathrm{H}_{\mathrm{ISCl}}$, but in a tree-like form, and with a significant use of cut.

\subsection{Admissibility results}

Due to the fact that we have relaxed the form of axioms accepted $((2.2)$ instead of (2.1), see page 270), there is no guarantee that the resulting system will be cut-free. This result will be proved below.

We refer to formulas specified in the premisses of a rule schema as active and to those specified in the conclusion as principal. Following [7], by height of a derivation we mean the maximal number of successive applications of the logical rules of $\mathbf{G} \mathbf{3}_{\mathrm{ISCl}}$. Moreover, by:

$$
\vdash_{n} \phi
$$

we shall mean that the sequent $\phi$ is derivable in $\mathbf{G} \mathbf{3}_{\text {ISCl }}$ with height no greater than $n$.

The terms of cut-height and formula weight defined below are used in proving admissibility of structural rules and follow the definitions from [7]. 
DEFInITION 6 (Cut-height). The cut-height of an application of the cut rule in a derivation $\mathcal{D}$ is the sum of heights of derivations of two premisses of cut.

Definition 7 (Formula weight). The weight is a function from the set of all formulas of $\mathcal{L}_{\mathrm{ISCl}}$ to the set of natural numbers, which fulfils the following conditions:

1. $w(\perp)=0$,

2. $w\left(p_{i}\right)=1$, for each $p_{i} \in V$,

3. $w(A \otimes B)=w(A)+w(B)+1$, where $\otimes \in\{\approx, \vee, \wedge, \rightarrow\}$.

Theorem 4 (Admissibility of weakening). If $\vdash_{n} \Gamma \Rightarrow C$, then $\vdash_{n} A, \Gamma \Rightarrow C$.

PRoof: A very straightforward proof relies on the observation that one can always transform a given derivation of $\Gamma \Rightarrow C$ into a derivation of $A, \Gamma \Rightarrow C$ by adding a formula $A$ to the antecedent of each sequent in the original derivation.

LEMma 6 (Height-preserving invertibility).

1. If $\vdash_{n} A \wedge B, \Gamma \Rightarrow C$, then $\vdash_{n} A, B, \Gamma \Rightarrow C$.

2. If $\vdash_{n} A \vee B, \Gamma \Rightarrow C$, then $\vdash_{n} A, \Gamma \Rightarrow C$ and $\vdash_{n} B, \Gamma \Rightarrow C$.

3. If $\vdash_{n} A \supset B, \Gamma \Rightarrow C$, then $\vdash_{n} B, \Gamma \Rightarrow C$.

4. If $\vdash_{n} \Gamma \Rightarrow C$, then $\vdash_{n} A \approx A, \Gamma \Rightarrow C$.

5. If $\vdash_{n} A \approx B, \Gamma \Rightarrow C$, then $\vdash_{n} A, A \approx B, \Gamma \Rightarrow C$.

6. If $\vdash_{n} A \approx B, C \approx D, \Gamma \Rightarrow E$, then $\vdash_{n}(A \otimes C) \approx(B \otimes D), A \approx$ $B, C \approx D, \Gamma \Rightarrow E$.

7. If $\vdash_{n} A \approx B, \Gamma \Rightarrow C$, then $\vdash_{n}(A \otimes A) \approx(B \otimes B), A \approx B, \Gamma \Rightarrow C$.

Proof: The argument for clauses 1.-3. is essentially the same as in the classical case and we skip it (see [7], if necessary). In the case of clauses 4.-7., each clause holds due to the admissibility of weakening. The second identity rule, $L_{\approx}^{2}$, is invertible only with respect to the right premiss.

Theorem 5 (Height-preserving admissibility of contraction). If $\vdash_{n} A, A, \Gamma \Rightarrow C$, then $\vdash_{n} A, \Gamma \Rightarrow C$.

Proof: By induction on the height of derivation. Assume $n=0$. Then the sequent $A, A, \Gamma \Rightarrow C$ is (i) an axiom or (ii) a conclusion of $L_{\perp}$. Naturally, in these cases sequent $A, \Gamma \Rightarrow C$ is an axiom or a conclusion of $L_{\perp}$.

Assume that the theorem holds up to $n$, and let $\vdash_{n+1} A, A, \Gamma \Rightarrow C$. If the contraction formula $A$ is not principal in the last applied rule $R$ of 
a given derivation, then we have to consider two cases: either $R$ is a onepremiss rule or a two-premisses rule. When the former is the case we have to consider the following situation:

$$
\frac{\vdash_{n} A, A, \Gamma^{\prime} \Rightarrow \Delta^{\prime}}{\vdash_{n+1} A, A, \Gamma \Rightarrow \Delta} R
$$

By the inductive hypothesis we have that $\vdash_{n} A, \Gamma^{\prime} \Rightarrow \Delta^{\prime}$. By applying $R$ to this sequent we obtain $\vdash_{n+1} A, \Gamma \Rightarrow \Delta$.

Similarly, if $R$ is a two-premisses rule:

$$
\frac{\vdash_{n} A, A, \Gamma^{\prime} \Rightarrow \Delta^{\prime} \quad \vdash_{n} A, A, \Gamma^{\prime \prime} \Rightarrow \Delta^{\prime \prime}}{\vdash_{n+1} A, A, \Gamma \Rightarrow \Delta} R
$$

then we apply the inductive hypothesis to the two premisses of $R$ in order to obtain (by means of $R$ ) the sequent $A, \Gamma \Rightarrow \Delta$ provable with height at most $n+1$.

If one of the contraction formulas is principal, then we have three cases where the formula is not an identity. Let us consider only implication.

$$
\frac{\vdash_{n} A \supset B, A \supset B, \Gamma \Rightarrow A \quad \vdash_{n} A \supset B, B, \Gamma \Rightarrow C}{\vdash_{n+1} A \supset B, A \supset B, \Gamma \Rightarrow C} L_{\supset}
$$

By the inductive hypothesis applied to the left premiss we know that

$$
\vdash_{n} A \supset B, \Gamma \Rightarrow A \text {. }
$$

For the right premiss we apply clause 3 . of Theorem 6 which yields that $\vdash_{n} B, B, \Gamma \Rightarrow C$. Now the inductive hypothesis can be applied, which results in $B, \Gamma \Rightarrow C$ being provable with height at most $n$. Application of $L_{\supset}$ to $(2.3)$ and $B, \Gamma \Rightarrow C$ gives us

$$
\vdash_{n+1} A \supset B, \Gamma \Rightarrow C
$$

The only non-standard cases are when the contracted formula is an equation, and the last rule used is one of $L_{\approx}^{2}, L_{\approx}^{3}$ or $L_{\approx}^{3 *}$. Let us consider the case when the last rule applied is $L_{\approx}^{2}$ :

$$
\frac{\vdash_{n} A \approx B, A \approx B, \Gamma \Rightarrow B \quad \vdash_{n} A, A \approx B, A \approx B, \Gamma \Rightarrow C}{\vdash_{n+1} A \approx B, A \approx B, \Gamma \Rightarrow C} L_{\approx}^{2}
$$

By inductive hypothesis, $\vdash_{n} A \approx B, \Gamma \Rightarrow B$ and $\vdash_{n} A, A \approx B, \Gamma \Rightarrow C$. We apply the rule $L_{\approx}^{2}$, to conclude $A \approx B, \Gamma \Rightarrow C$ in at most $n+1$ steps: 


$$
\frac{\vdash_{n} A \approx B, \Gamma \Rightarrow B \quad A, A \approx B, \Gamma \Rightarrow C}{\vdash_{n+1} A \approx B, \Gamma \Rightarrow C} L_{2}
$$

Due to the fact that in one of the rules of the system two formulas are principal $\left(L_{\approx}^{3}\right)$, we have to consider a situation, where both contraction formulas are principal.

$$
\frac{\vdash_{n}(B \otimes B) \approx(C \otimes C), B \approx C, B \approx C, \Gamma \Rightarrow \Delta}{\vdash_{n+1} B \approx C, B \approx C, \Gamma \Rightarrow \Delta} L_{\approx}^{3}
$$

By inductive hypothesis applied to the premiss we get $\vdash_{n}(B \otimes B) \approx$ $(C \otimes C), B \approx C, \Gamma \Rightarrow \Delta$. Now an application of the rule $L_{\approx}^{3 *}$, results in $B \approx C, \Gamma \Rightarrow \Delta$ with at most $n+1$ steps:

$$
\frac{\vdash_{n}(B \otimes B) \approx(C \otimes C), B \approx C, \Gamma \Rightarrow \Delta}{\vdash_{n+1} B \approx C, \Gamma \Rightarrow \Delta} L_{\approx}^{3 *}
$$

This case clearly shows how the rule obtained by the closure condidtion is necessary for proving admissibility of contraction.

THEOREM 6. The cut rule

$$
\frac{\Gamma^{\prime} \Rightarrow D \quad D, \Gamma^{\prime \prime} \Rightarrow C}{\Gamma^{\prime}, \Gamma^{\prime \prime} \Rightarrow C} \text { cut }
$$

is admissible in $\mathbf{G} \mathbf{3}_{\mathrm{ISCl}}$.

Proof: The proof is organized as in [6]. The idea is to divide all the cases to consider into some classes. The first class enhances the cases where at least one of the premisses of the cut-rule is an axiom or a conclusion of $L_{\perp}$. Assume it is the left premiss. Then (the case of an axiom) $D$ is a propositional variable or an equation and it belongs to $\Gamma^{\prime}$. In this case cut can be completely eliminated by (possibly multiple) application(s) of weakening:

$$
\frac{D, \Gamma^{\prime \prime} \Rightarrow C}{\Gamma^{\prime}, \Gamma^{\prime \prime} \Rightarrow C} L_{w}
$$

If $\perp$ occurs in $\Gamma^{\prime}$ (the case of $L_{\perp}$ ), then the conclusion of cut, that is, $\Gamma^{\prime}, \Gamma^{\prime \prime} \Rightarrow C$, also follows by $L_{\perp}$, so the application of the cut rule can be eliminated. 
Similar simplifications can be applied, if we assume that the right premiss was one of the axioms or a conclusion of $\perp$. There is one subtlety here. Assume $D=\perp$. Thus we arrive at:

$$
\frac{\Gamma^{\prime} \Rightarrow \perp \quad \perp, \Gamma^{\prime \prime} \Rightarrow C}{\Gamma^{\prime}, \Gamma^{\prime \prime} \Rightarrow C} \text { cut }
$$

If $\perp$ occurs in $\Gamma^{\prime}$ or $\Gamma^{\prime \prime}$, then cut can be eliminated. But if it does not occur in $\Gamma^{\prime}$, we have to consider the rule which was applied in order to obtain the left premiss. In fact, this case follows under class $\mathbf{I}$ of cases considered below, so there is no need to consider it separately.

All the other cases (i.e. those where a premiss is neither an axiom nor a conclusion of $L_{\perp}$ ) can be divided into the following three classes.

I The cut formula $D$ is not principal in the left premiss. We consider only the cases where the left premiss is itself a conclusion of a $\approx$-specific rules.

(1) The last rule applied was $L_{\approx}^{1}$. Cut-height equals $(m+1)+m^{\prime}$ :

$$
\begin{aligned}
& \text { m }
\end{aligned}
$$

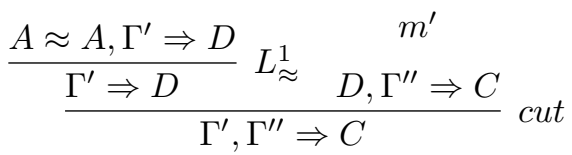

This derivation is transformed into a derivation of smaller cut-height (equal to $m+m^{\prime}$ ):

$$
\frac{A \approx A, \Gamma^{\prime} \Rightarrow D \quad D, \Gamma^{\prime \prime} \Rightarrow C}{\frac{A \approx A, \Gamma^{\prime}, \Gamma^{\prime \prime} \Rightarrow C}{\Gamma^{\prime}, \Gamma^{\prime \prime} \Rightarrow C}} \text { cut } L_{\approx}^{1}
$$

(2) The last rule applied was $L_{\approx}^{2}$. The cut-height equals $(\max (m, n)+1)$ $+m^{\prime}$ :

$m$

$n$

$$
\frac{A \approx B, \Gamma^{\prime} \Rightarrow B \quad A, A \approx B, \Gamma^{\prime} \Rightarrow D}{\frac{A \approx B, \Gamma^{\prime} \Rightarrow D}{A \approx B, \Gamma^{\prime}, \Gamma^{\prime \prime} \Rightarrow C}} L_{\approx}^{2} \quad \begin{gathered}
m^{\prime} \\
\frac{D, \Gamma^{\prime \prime} \Rightarrow C}{c u t}
\end{gathered}
$$


This derivation is transformed into derivation with cut of cut-height $n+m^{\prime}$ :

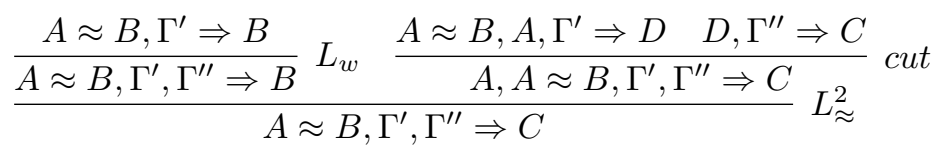

(3) The last rule applied was $L_{\approx}^{3}$. Cut-height equals $(m+1)+m^{\prime}$ :

$m$

$$
\frac{\left(A \otimes C^{*}\right) \approx\left(B \otimes D^{*}\right), A \approx B, C^{*} \approx D^{*}, \Gamma^{\prime} \Rightarrow D}{\frac{A \approx B, C^{*} \approx D^{*}, \Gamma^{\prime} \Rightarrow D}{A \approx B, C^{*} \approx D^{*}, \Gamma^{\prime}, \Gamma^{\prime \prime} \Rightarrow C}} \begin{gathered}
m^{\prime} \\
\frac{D}{2}, \Gamma^{\prime \prime} \Rightarrow C \\
\text { cut }
\end{gathered}
$$

This derivation is transformed into a derivation with a lesser cut-height $\left(m+m^{\prime}\right)$ :

$$
\frac{\left(A \otimes C^{*}\right) \approx\left(B \otimes D^{*}\right), A \approx B, C^{*} \approx D^{*}, \Gamma^{\prime} \Rightarrow D \quad D, \Gamma^{\prime \prime} \Rightarrow C}{\frac{\left(A \otimes C^{*}\right) \approx\left(B \otimes D^{*}\right), A \approx B, C^{*} \approx D^{*}, \Gamma^{\prime}, \Gamma^{\prime \prime} \Rightarrow C}{A \approx B, C^{*} \approx D^{*}, \Gamma^{\prime}, \Gamma^{\prime \prime} \Rightarrow C} L_{\approx}^{3}} \text { cut }
$$

(4) The last rule applied was $L_{\approx}^{3 *}$. Cut-height equals $(m+1)+m^{\prime}$ :

$m$

$$
\frac{(A \otimes A) \approx(B \otimes B), A \approx B, \Gamma^{\prime} \Rightarrow D}{\frac{A \approx B, \Gamma^{\prime} \Rightarrow D}{A \approx}} L_{\approx}^{3 *} \quad \begin{gathered}
m^{\prime} \\
A \approx B, \Gamma^{\prime \prime} \Rightarrow C
\end{gathered} \text { cut }
$$

This derivation is transformed into a derivation with a lesser cut-height $\left(m+m^{\prime}\right)$ :

$$
\frac{(A \otimes A) \approx(B \otimes B), A \approx B, \Gamma^{\prime} \Rightarrow D \quad D, \Gamma^{\prime \prime} \Rightarrow C}{\frac{(A \otimes A) \approx(B \otimes B), A \approx B, \Gamma^{\prime}, \Gamma^{\prime \prime} \Rightarrow C}{A \approx B, \Gamma^{\prime}, \Gamma^{\prime \prime} \Rightarrow C} L_{\approx}^{3 *}} \text { cut }
$$

II When the cut-formula is principal in the left premiss only, we consider the last rule applied to the right premiss of cut. Note that in this case the 
cut formula cannot be an equation, due to the fact that there are no right identity rules. The transformations are analogous to the ones in [7].

III If the cut formula $D$ is principal in both premisses, only classical rules can be applied to $D$.

\section{Discussion}

\subsection{Subformula property}

Note that in the described system subformula property is not entailed by cut elimination due to the non-analytic character of the identity rules. Nevertheless, the notion of a subformula can be modified as follows.

Definition 8. Let $F$ be a formula of $\mathcal{L}_{\mathrm{ISCl}}$. The set of subformulas of $F$, in symbols, $\operatorname{sub}(F)$, is the smallest set satisfying the following conditions:

- $F \in \operatorname{sub}(F)$;

- if $F$ is of the form $B \otimes C$, then $\operatorname{sub}(B) \subseteq \operatorname{sub}(B \otimes C)$ and $\operatorname{sub}(C) \subseteq$ $\operatorname{sub}(B \otimes C)$.

Moreover, the set $\operatorname{sub}(F)$ is closed under the following rules:

$$
\begin{gathered}
\frac{A \approx C \in \operatorname{sub}(F) \quad B \approx D \in \operatorname{sub}(F)}{\operatorname{sub}((A \otimes B) \approx(C \otimes D)) \subseteq \operatorname{sub}(F)} \\
\frac{A \in \operatorname{sub}(F)}{A \approx A \in \operatorname{sub}(F)}
\end{gathered}
$$

Let $d$ be a derivation in $\mathbf{G} \mathbf{3}_{\mathrm{ISCl}}$. By labels $(d)$ we denote the set of all formulas occurring in sequents labelling nodes of $d$. Now we can state:

TheOREM 7. If a sequent $\Rightarrow A$ is provable, then there exists a proof $d$ of $\Rightarrow A$, such that

$$
\text { labels }(d) \subseteq \operatorname{sub}(A)
$$

Note that this result cannot be extended to derivations which are not proofs, due to the lack of restrictions imposed on the first identity rule. 


\subsection{Variants of the calculus}

Note that the second $\mathrm{SCl}$ axiom can be turned into a rule in such a way that intuitionistic implication occurs explicitly in the premiss:

$$
\frac{A \approx B, B \supset A, \Gamma \Rightarrow C}{A \approx B, \Gamma \Rightarrow C} a L_{\approx}^{2}
$$

In a system in which we exchange $L_{\approx}^{2}$ with $a L_{\approx}^{2}$ the first rule becomes derivable:

$$
\frac{\frac{A \approx B, \Gamma \Rightarrow B}{A \approx B, B \supset A, \Gamma \Rightarrow B} L_{w} \quad A, A \approx B, \Gamma \Rightarrow C}{\frac{A \approx B, B \supset A, \Gamma \Rightarrow C}{A \approx B, \Gamma \Rightarrow C} a L^{2}} L_{\supset}
$$

On the other hand, $a L_{\approx}^{2}$ cannot be derived in a system with $L_{\approx}^{2}$, since the latter is analytic, while the former introduces new formula $B \supset A$. In both this systems the rule of cut is admissible.

In our strategy of building a sequent calculus for ISCI we kept close to the syntactic structure of the identity axioms, as they are expressed in the Hilbert-style system. Another strategy can be applied in order to obtain a different system. This time we make use of the analogy between propositional and term identity.

We have to assume that:

- identity is reflexive, and

- identical propositions can be exchanged in arbitrary contexts, and

- identity is stronger than intuitionistic equivalence.

These assumptions can be transformed into rules in the following way:

$$
\begin{gathered}
\frac{A \approx A, \Gamma \Rightarrow C}{\Gamma \Rightarrow C} \text { ref } \quad \frac{D_{B}^{A}, \Gamma \Rightarrow C}{A \approx B, D, \Gamma \Rightarrow C} \text { rep } \\
\frac{A \approx B, B \supset A, \Gamma \Rightarrow C}{A \approx B, \Gamma \Rightarrow C} a L_{\approx}^{2}
\end{gathered}
$$

where $D_{B}^{A}$ is the result of replacing $A$ with $B$ in some (possibly all) contexts. Let us note that each identity axiom can be derived in the system. Let us prove the fourth axiom: 


$$
\begin{gathered}
\frac{(A \otimes B) \approx(C \otimes D) \Rightarrow(A \otimes B) \approx(C \otimes D)}{(A \otimes B) \approx(C \otimes B), B \approx D \Rightarrow(A \otimes B) \approx(C \otimes D)} \text { rep } \\
\frac{(A \otimes B) \approx(A \otimes B), A \approx C, B \approx D \Rightarrow(A \otimes B) \approx(C \otimes D)}{A \approx} r e p \\
\frac{A \approx C, B \approx D \Rightarrow(A \otimes B) \approx(C \otimes D)}{\Rightarrow((A \approx C) \wedge(B \approx D)) \supset((A \otimes B) \approx(C \otimes D))} L_{\wedge}, R_{\supset}
\end{gathered}
$$

Let us also note that the rule of contraction is not height-preserving admissible in this system due to the shape of the replacement rule. This however can be fixed up by replacing rep by the following, more general version:

$$
\frac{D_{B}^{A}, A \approx B, D, \Gamma \Rightarrow C}{A \approx B, D, \Gamma \Rightarrow C} r e p^{*}
$$

The rule of cut is admissible in the system and the argument is very similar to the one presented in Section 2.3.

Let us also note that each rule of our initial system $\mathbf{G} \mathbf{3}_{\mathrm{ISCl}}$ can be derived in the system we have just defined - the new one is thus more general. Here is a derivation of $L_{\approx}^{3}(Z$ stands for $(A \otimes B) \approx(C \otimes D))$ :

$$
\begin{gathered}
\frac{Z, A \approx C, B \approx D, \Gamma \Rightarrow E}{\frac{Z,(A \otimes B) \approx(C \otimes B),(A \otimes B) \approx(A \otimes B), A \approx C, B \approx D, \Gamma \Rightarrow E}{Z,(A \otimes B) \approx(A \otimes B), A \approx C, B \approx D, \Gamma \Rightarrow E}} r_{w}{ }_{e}{ }^{*} p^{*} \\
\frac{Z, A \approx C, B \approx D, \Gamma \Rightarrow E}{A \approx C, B \approx D, \Gamma \Rightarrow E} \text { ref }
\end{gathered}
$$

The rule $L_{\approx}^{3 *}$ can be derived by the same mechanism (with the use of weakening). $L_{\approx}^{2}$ is also derivable, in exactly the same manner as is shown at the beginning of this section.

The problem is that the approach now is semantical - we know that $\approx$ denotes identity, thus we can construct the rules. Therefore the described approach is not mechanical and strongly depends on our ability to interpret the corresponding axioms. 


\section{Conclusions}

We showed that Suszko's propositional identity connective has a natural constructive interpretation. Therefore, the logic $\mathrm{ISCl}$ can be considered as a legitimate (in the sense of the underlying philosophical intuitions) extension of intuitionistic logic. We defined possible world semantics for $\mathrm{ISCl}$ along with two cut-free sequent calculi for ISCI.

The future work will cover the construction and the analysis of natural deduction system for ISCI along with the typed lambda calculus corresponding to it, which will put more light on the constructive interpretation of Suszko's propositional identity connective.

\section{References}

[1] S. L. Bloom and R. Suszko, Investigations into the sentential calculus with identity, Notre Dame Journal of Formal Logic, Vol. 13, No. 3 (1972), pp. 289-308.

[2] J. G. Granström, Treatise on intuitionistic type theory, Springer Science \& Business Media, Dordrecht, 2011.

[3] J. R. Hindley, Basic simple type theory, Cambridge University Press, Cambridge, 1997.

[4] S. C. Kleene, Introduction to Metamathematics, Amsterdam: NorthHolland Publishing Co.; Groningen: P. Noordhoff N.V., 1952.

[5] P. Lukowski, Intuitionistic sentential calculus with identity, Bulletin of the Section of Logic, Vol. 19, No. 3 (1990), pp. 92-99.

[6] S. Negri and J. von Plato, Cut elimination in the presence of axioms, Bulletin of Symbolic Logic, Vol. 4, No. 04 (1998), pp. 418-435.

[7] S. Negri and J. von Plato, Structural Proof Theory, Cambridge University Press, Cambridge, 2001.

[8] S. Negri and J. von Plato, Proof Analysis: a Contribution to Hilbert's Last Problem, Cambridge University Press, Cambridge, 2011.

[9] S. Negri, J. von Plato, and T. Coquand, Proof-theoretical analysis of order relations, Archive for Mathematical Logic, Vol. 43, No. 3 (2004), pp. 297-309.

[10] R. Suszko, Abolition of the Fregean axiom, [in:] R. Parikh (ed.), Logic Colloquium, pp. 169-239, Berlin, Heidelberg, 1975, Springer. 
[11] A. S. Troelstra and H. Schwichtenberg, Basic Proof Theory, Camridge University Press, Cambridge, second edition, 2000.

[12] L. Viganò, Labelled non-classical logics, Kluwer Academic Publishers, Boston, 2000.

Adam Mickiewicz University

Faculty of Psychology and Cognitive Science

Department of Logic and Cognitive Science

e-mail: szymon.chlebowski@amu.edu.pl

e-mail: dorota.leszczynska@amu.edu.pl 